\title{
N-ARY MAXIMUM LIKELIHOOD TARGET DETECTION WITH TIME REVERSAL
}

\author{
Foroohar Foroozan and Amir Asif \\ Department of Computer Science and Engineering \\ York University, Toronto, ON, Canada \\ Email: \{foroozan, asif\}@cse.yorku.ca
}

\begin{abstract}
A time reversal (TR) device has the capability of receiving a time varying signal, saving it in memory, and retransmitting the stored signal back into the medium in the reversed direction of time. The paper designs an $\mathrm{N}$-ary maximum likelihood TR detector capable of detecting the presence of a target and classifying the detected target as an unknown enemy target or one of the $(N-2)$ known friendly targets. In our experiments, the proposed $N$-ary TR detector provides a gain of over $5 \mathrm{~dB}$ over conventional detectors at low signal to noise ratios (SNR).
\end{abstract}

Index Terms - Time Reversal, Detection, Neyman Pearson test, Maximum Likelihood, and Green's function.

\section{INTRODUCTION}

Time reversal (TR) [1] offers an attractive alternative to traditional detectors, e.g., the matched filter in one dimensional signal processing and the matched field processing (MFP) [2] in multidimensions. The difficulty with MFP is that the Green's function used to model the channel is not known and has to be computed numerically. By its inherent nature, TR intrinsically derives the Green's function of the channel as long as the transducer array provides an adequate sampling of the channel. A second advantage of TR lies in the positive treatment of the channel multipaths, which are typically considered a detrimental in traditional detectors.

The paper derives an $N$-ary maximum likelihood detector

$$
\begin{aligned}
\mathbb{H}_{0}: & \text { No target is present. } \\
\mathbb{H}_{i}: & \text { Friendly target } i,(1 \leq i \leq(N-2)), \text { is present. } \\
\mathbb{H}_{N-1}: & \text { Enemy target, } i=(N-1), \text { is present. }
\end{aligned}
$$

using time reversal in a rich cluttering environment with a random velocity profile. In other words, we are interested in detecting the presence of a target and classifying the detected target as an unknown enemy target or one of the known friendly targets. Our algorithm is based on the Neyman Pearson test and extends the framework presented in [3], where the binary detection problem is considered, to multiple targets $(1 \leq i \leq(N-1))$. We compare the performance of our $N$-ary TR detection algorithm with conventional detectors that are based on forward propagation and do not include the time reversal step. In our simulations, the TR detector provides a gain of $5 \mathrm{~dB}$ over conventional detectors at low signal to noise ratios (SNR).

The paper is organized as follows. Section 2 defines the notation in terms of the four steps (forward propagation, forward reflection,

This work was supported in part by the Natural Science and Engineering Research Council (NSERC), Canada under Grant No. 228415-2007.
TR propagation, and TR reflection) involved in the $N$-ary TR detector. Sections 3 and 4 derive the maximum likelihood (ML) functions for the conventional and TR detectors in a multiple target environment. Section 5 compares the performance of the $N$-ary TR detector with conventional detectors, which use only the forward propagation and reflection steps. Section 6 concludes the paper.

\section{NOTATION}

Consider a spatial domain (channel) embedded with target $i,(1 \leq$ $i \leq(N-1))$. Our algorithm detects which target $i$ is present using the following steps.

1. Forward Propagation: A single transceiver at $\vec{y}_{p}$ probes the channel with a wideband signal $f[k]$ of duration $(0 \leq k \leq$ $(K-1)), k$ being the time index.

2. Forward Reflection: Target $i$ at $\vec{y}_{t_{i}}$ reflects the transmitted signal such that the field observed at the transceiver is given by $g_{i}\left[\vec{y}_{p}, \vec{y}_{t_{i}}, k\right]$. In terms of the impulse response or the target signature, $h_{t_{i}}[k]$, the observed field for target $i,(1 \leq i \leq$ $(N-1))$, is given by

$$
g_{i}\left[\vec{y}_{p}, \vec{y}_{t_{i}}, k\right]=f[k] \circledast\left(h_{t_{i}}[k]+h_{c}[k]\right)+v_{i}[k],
$$

where $\circledast$ denotes convolution and $v_{i}[k]$ is zero mean, circular white Gaussian noise with variance $\sigma_{v}^{2}$, denoted as $\mathcal{C N}\left(0, \sigma_{v}^{2}\right)$. Clutter $\left(f[k] \circledast h_{c}[k]\right)$ is subtracted from Eq. (1) using the background subtraction process described later in Section 2.1.

3. TR Propagation: Following the principle of TR, the received signal $g_{i}\left[\vec{y}_{p}, \vec{y}_{t_{i}}, k\right]$ is energy normalized, time reversed, and retransmitted back into the medium. The retransmitted signal is given by $c_{i} g_{i}\left[\vec{y}_{p}, \vec{y}_{t_{i}}, K-k\right]$, where $c_{i}$ is the normalization constant.

4. TR Reflection: The TR signal is reflected by the target and the field observed at the transceiver is given by

$$
\begin{aligned}
p_{i}\left[\vec{y}_{p}, \vec{y}_{t_{i}}, k\right] & =c_{i} g_{i}\left[\vec{y}_{p}, \vec{y}_{t_{i}}, K-k\right] \\
& \circledast\left(h_{t_{i}}[k]+h_{c}[k]\right)+w_{i}[k]
\end{aligned}
$$

with $w_{i}[k]$ being $\mathcal{C N}\left(0, \sigma_{w}^{2}\right)$. As in Step 2, we subtract clutter from Eq. (2) using the background subtraction step outlined in Section 2.1.

Steps 1-4 are repeated $M$ times, so we have $M$ observations from Steps 2 and 4. After subtracting clutter and introducing superscript $m$ to denote the $m$ 'th observation, Eq. (1) is modified as

$$
g_{i}^{(m)}\left[\vec{y}_{p}, \vec{y}_{t_{i}}, k\right]=f[k] \circledast h_{t_{i}}[k]+v_{i}^{(m)}[k],
$$


or, equivalently in the discretized frequency domain as

$$
\begin{aligned}
& \forall q=0, \cdots, Q-1 \text { and } m=1, \cdots, M, \\
& \quad G_{i}^{(m)}\left(\omega_{q}\right)=F\left(\omega_{q}\right) H_{t_{i}}\left(\omega_{q}\right)+V_{i}^{(m)}\left(\omega_{q}\right),
\end{aligned}
$$

where the discretized frequency $\omega_{q}=2 q \pi / Q,(0 \leq q \leq Q-$ 1) and $G_{i}^{(m)}\left(\omega_{q}\right)$ is the discrete Fourier transform (DFT) of $g_{i}^{(m)}\left[\vec{y}_{p}, \vec{y}_{t_{i}}, k\right]$. Similarly, we use uppercase letters $F\left(\omega_{q}\right), H_{t_{i}}\left(\omega_{q}\right)$ and $V_{i}^{(m)}\left(\omega_{q}\right)$ to represent the DFT's of the time domain variables $f[k], h_{t_{i}}[k]$ and $v_{i}^{(m)}[k]$. Likewise, for multiple observations, Eq. (2) in Step 4 is given by

$$
\begin{aligned}
p_{i}^{(m)} & \left.\vec{y}_{p}, \vec{y}_{t_{i}}, k\right]= \\
& c_{i}^{(m)} g_{i}^{(m)}\left[\vec{y}_{p}, \vec{y}_{t_{i}}, K-k\right] \circledast h_{t_{i}}[k]+w_{i}^{(m)}[k]
\end{aligned}
$$

Substituting the value of $g_{i}^{(m)}\left[\vec{y}_{p}, \vec{y}_{t_{i}}, K-k\right]$ in Eq. (5) from Eq. (3) and taking the DFT, we get

$$
\begin{aligned}
\forall q=0, \cdots, Q & -1 \text { and } m=1, \cdots, M, \\
P_{i}^{(m)}\left(\omega_{q}\right) & =c_{i}^{(m)} F^{*}\left(\omega_{q}\right)\left|H_{t_{i}}\left(\omega_{q}\right)\right|^{2} \\
& +c_{i}^{(m)} H_{t_{i}}\left(\omega_{q}\right) V_{i}^{(m)}{ }^{*}\left(\omega_{q}\right)+W_{i}^{(m)}\left(\omega_{q}\right)
\end{aligned}
$$

where $P_{i}^{(m)}\left(\omega_{q}\right)$ and $W_{i}^{(m)}\left(\omega_{q}\right)$ are respectively the DFT's of the time domain variables $p_{i}^{(m)}\left[\vec{y}_{p}, \vec{y}_{t_{i}}, k\right]$ and $w_{i}^{(m)}[k]$. Superscript $*$ denotes conjugation. Our $N$-ary TR detector uses observations from Step 4 (Eq. (6)) to detect which target $i$ is present. The conventional detector is based on Eq. (4) and does not require Steps 3 and 4.

Vector Notation: Using the standard vector notation

$$
\begin{aligned}
\mathbf{G}_{i}^{(m)} & =\left[G_{i}^{(m)}\left(\omega_{0}\right), \ldots, G_{i}^{(m)}\left(\omega_{Q-1}\right)\right]^{T} \\
\mathbf{P}_{i}^{(m)} & =\left[P_{i}^{(m)}\left(\omega_{0}\right), \ldots, P_{i}^{(m)}\left(\omega_{Q-1}\right)\right]^{T} \\
\mathbf{H}_{t_{i}} & =\left[H_{t_{i}}\left(\omega_{0}\right), \ldots, H_{t_{i}}\left(\omega_{Q-1}\right)\right]^{T} \\
\mathbf{V}_{i}^{(m)} & =\left[V_{i}^{(m)}\left(\omega_{0}\right), \ldots, V_{i}^{(m)}\left(\omega_{Q-1}\right)\right]^{T} \\
\text { and } \quad \mathbf{W}_{i}^{(m)} & =\left[W_{i}^{(m)}\left(\omega_{0}\right), \ldots, W_{i}^{(m)}\left(\omega_{Q-1}\right)\right]^{T}
\end{aligned}
$$

to collect respectively the DFT values $G_{i}^{(m)}\left(\omega_{q}\right), P_{i}^{(m)}\left(\omega_{q}\right)$, $H_{t_{i}}\left(\omega_{q}\right), V_{i}^{(m)}\left(\omega_{q}\right)$, and $W_{i}^{(m)}\left(\omega_{q}\right)$ obtained from the $m$ 'th observation, Eqs. (4) and (6) are given by

$$
\begin{aligned}
\mathbf{G}_{i}^{(m)} & =\mathbf{F H}_{t_{i}}+\mathbf{V}_{i}^{(m)} \\
\mathbf{P}_{i}^{(m)} & =c_{i}^{(m)} \mathbf{G}_{i}^{(m)^{*}} \odot \mathbf{H}_{t_{i}}+\mathbf{W}_{i}^{(m)}
\end{aligned}
$$

where $\mathbf{F}$ is a diagonal matrix of order $Q$ with the DFT values $F\left(\omega_{q}\right)$ arranged along its diagonal and symbol $\odot$ denotes the Hadamard product. Note that $\mathbf{V}_{i}^{(m)} \sim \mathcal{C N}\left(\underline{0}, \sigma_{v}^{2} \mathbf{I}_{Q}\right)$ and $\mathbf{W}_{i}^{(m)} \sim \mathcal{C N}\left(\underline{0}, \sigma_{w}^{2} \mathbf{I}_{Q}\right)$, which leads to the test statistics

$$
\begin{aligned}
& \mathbf{G}_{i}^{(m)} \mid \mathbb{H}_{i} \sim \mathcal{C N}\left(\mathbf{F} \mathbf{H}_{t_{i}}, \sigma_{v}^{2} \mathbf{I}_{Q}\right) \\
& \mathbf{P}_{i}^{(m)} \mid \mathbb{H}_{i} \sim \mathcal{C N}\left(c_{i}^{(m)} \mathbf{F}^{*} \mathbf{H}_{t_{i}}^{*} \odot \mathbf{H}_{t_{i}},\right. \\
&\left.\sigma_{v}^{2} c_{i}^{(m)}{ }^{2} \operatorname{diag}\left\{\mathbf{H}_{t_{i}}^{*} \odot \mathbf{H}_{t_{i}}\right\}+\sigma_{w}^{2} \mathbf{I}_{Q}\right)
\end{aligned}
$$

that are both circular Gaussian. The statistics for hypothesis $\mathbb{H}_{0}$ are obtained by substituting $\mathbf{H}_{t_{i}}=0$ in Eqs. (9) and (10). Finally, the $M$ observations $\mathbf{G}_{i}^{(m)}$ and $\mathbf{P}_{i}^{(m)}$ are stacked in $(Q M \times 1)$ vectors $\mathbf{G}_{\mathbf{i}}$ and $\mathbf{P}_{\mathbf{i}}$ resulting in

$$
\begin{aligned}
\mathbf{G}_{\mathbf{i}}= & \mathbf{1} \otimes \mathbf{F H}_{t_{i}}+\mathbf{V}_{i} \\
\mathbf{P}_{\mathbf{i}}= & \mathbf{c}_{\mathbf{i}} \otimes\left[\mathbf{F}^{*} \mathbf{H}_{t_{i}}^{*} \odot \mathbf{H}_{t_{i}}\right] \\
& +\left(\mathbf{c}_{\mathbf{i}} \otimes \mathbf{H}_{t_{i}}\right) \odot \mathbf{V}_{i}^{*}+\mathbf{W}_{i}
\end{aligned}
$$

where $\mathbf{c}_{i}=\left[c_{i}^{(1)}, \ldots, c_{i}^{(M)}\right]^{T}, \mathbf{1}$ is an $(M \times 1)$ vector with all unit entries, symbol $\otimes$ denotes the Kronecker product, $\mathbf{V}_{\mathbf{i}}$ and $\mathbf{W}_{\mathbf{i}}$ are zero mean, complex Gaussian random vectors, representing noise $\mathbf{V}_{i}^{(m)}$ and $\mathbf{W}_{i}^{(m)}$, respectively. The statistics of $\mathbf{G}_{\mathbf{i}} \mid \mathbb{H}_{i}$ and $\mathbf{P}_{\mathbf{i}} \mid \mathbb{H}_{i}$ are given by

$$
\begin{aligned}
\mathbf{G}_{\mathbf{i}} \mid \mathbb{H}_{i} \sim & \mathcal{C N}\left(\mathbf{1} \otimes \mathbf{F H}_{t_{i}}, \sigma_{v}^{2} \mathbf{I}_{M} \otimes \mathbf{I}_{Q}\right) \\
\mathbf{P}_{\mathbf{i}} \mid \mathbb{H}_{i} \sim & \mathcal{C N}\left(\mathbf{c}_{\mathbf{i}} \otimes\left[\mathbf{F}^{*} \mathbf{H}_{t_{i}}^{*} \odot \mathbf{H}_{t_{i}}\right]\right. \\
& \left.\sigma_{v}^{2} \mathbf{C}_{\mathbf{i}}^{2} \otimes \operatorname{diag}\left\{\mathbf{H}_{t_{i}}^{*} \odot \mathbf{H}_{t_{i}}\right\}+\sigma_{w}^{2} \mathbf{I}_{M} \otimes \mathbf{I}_{Q}\right)
\end{aligned}
$$

where $\mathbf{C}_{i}=\operatorname{diag}\left\{\mathbf{c}_{i}\right\}$. The statistics of $\mathbf{G}_{0}$ and $\mathbf{P}_{0}$ under hypothesis $\mathbb{H}_{0}$ are obtained by substituting $\mathbf{H}_{t_{i}}=0$ in Eqs. (13) and (14).

\subsection{Clutter}

To subtract clutter from the reflected signal $g_{i}\left[\vec{y}_{p}, \vec{y}_{t_{i}}, k\right]$ in Eq. (1) and the TR reflected signal $p_{i}\left[\vec{y}_{p}, \vec{y}_{t_{i}}, k\right]$ in Eq. (2), a background subtraction step is introduced where the probing signal $f[k]$ is transmitted into the channel when no target is present. This training step is repeated $L$ times. Following the derivation of Eq. (4), the observations $G_{c}^{(l)}\left(\omega_{q}\right)$ in the absence of any target is given by

$$
\begin{aligned}
& \forall q=0, \cdots, Q-1 \text { and } l=1, \cdots, L, \\
& \quad G_{c}^{(l)}\left(\omega_{q}\right)=F\left(\omega_{q}\right) H_{c}\left(\omega_{q}\right)+U^{(l)}\left(\omega_{q}\right),
\end{aligned}
$$

where $H_{c}\left(\omega_{q}\right)$ is the clutter transfer function and $U^{(l)}\left(\omega_{q}\right) \sim$ $\mathcal{C N}\left(0, \sigma_{u}^{2}\right)$. Based on Eq. (15), the estimated clutter response $\widehat{H}_{c}\left(\omega_{q}\right)$ that minimizes the mean square error $\left[H_{c}\left(\omega_{q}\right)-\widehat{H}_{c}\left(\omega_{q}\right)\right]^{2}$ is given by

$$
\widehat{H}_{c}\left(\omega_{q}\right)=\frac{1}{L} F^{-1}\left(\omega_{q}\right) \sum_{l=1}^{L} G_{c}^{(l)}\left(\omega_{q}\right) .
$$

The above estimate is unbiased such that $\widehat{H}_{c}\left(\omega_{q}\right)$ converges to the true clutter response as the limit $L \rightarrow \infty$. In the subsequent discussion, we assume that the background subtraction step is used to remove clutter from both the reflected signal $g_{i}\left[\vec{y}_{p}, \vec{y}_{t_{i}}, k\right]$ and the TR reflected signal $p_{i}\left[\vec{y}_{p}, \vec{y}_{t_{i}}, k\right]$.

\section{N-ARY CONVENTIONAL ML DETECTOR}

The section derives the conventional ML detector based on the clutter suppressed reflected signal $g_{i}^{(m)}\left[\vec{y}_{p}, \vec{y}_{t_{i}}, k\right]$, or equivalently its vector equivalent DFT representation $\mathbf{G}_{i}$ in Eq. (13). We consider first the case where the enemy target response $\mathbf{H}_{t_{N-1}}$ is assumed known followed by the general case where $\mathbf{H}_{t_{N-1}}$ is unknown.

\subsection{Known Enemy Target Channel Response}

The target channel responses $\mathbf{H}_{t_{i}}$, for $(0 \leq i \leq(N-1))$, are consequently all known and the pdf's for $\mathbf{G}_{i}$ under hypotheses $\mathbb{H}_{i}$ and $\mathbb{H}_{0}$ are derived from Eq. (13) and are given by

$$
\begin{aligned}
\operatorname{Pr}\left(\mathbf{G}_{i} \mid \mathbb{H}_{i}\right) & =\prod_{m=1}^{M} \frac{1}{\pi^{Q}\left(\sigma_{v}^{2}\right)^{Q}} e^{-\frac{\| \mathbf{G}_{i}^{(m)}-\mathbf{F H}_{t_{i} \|^{2}}}{\sigma_{v}^{2}}} \\
\operatorname{Pr}\left(\mathbf{G}_{i} \mid \mathbb{H}_{0}\right) & =\prod_{m=1}^{M} \frac{1}{\pi^{Q}\left(\sigma_{v}^{2}\right)^{Q}} e^{-\frac{\left\|\mathbf{G}_{i}^{(m)}\right\|^{2}}{\sigma_{v}^{2}}}
\end{aligned}
$$

where observations $m,(1 \leq m \leq M)$, are assumed independent of each other. Notation $\|\cdot\|$ denotes the Frobenius norm. Eqs. (17) and (18) lead to the $\log$ likelihood ratio $\ell_{i}\left(\mathbf{G}_{i}\right)$ expressed in Theorem 1. 
Theorem 1. The test statistics $\ell_{i}\left(\mathbf{G}_{i}\right)$ of the conventional detector based on the observation vector $\mathbf{G}_{i}$ is given by

$$
\ell_{i}\left(\mathbf{G}_{i}\right)=\Re\left(\frac{\left(\mathbf{F H}_{t_{i}}\right)^{H} \sum_{m=1}^{M} \mathbf{G}_{i}^{(m)}}{\sigma_{v}\left\|\mathbf{F H}_{t_{i}}\right\|}\right)
$$

with the conditional probability density functions

$$
\begin{aligned}
\ell_{i}\left(\mathbf{G}_{i}\right) \mid \mathbb{H}_{0} & \sim \mathcal{C N}(0, M / 2) \\
\forall(i \neq 0), \quad \ell_{i}\left(\mathbf{G}_{i}\right) \mid \mathbb{H}_{i} & \sim \mathcal{C N}\left(\mu_{i}, M / 2\right)
\end{aligned}
$$

where the mean $\mu_{i}$ equals $M\left\|\mathbf{F} \mathbf{H}_{t_{i}}\right\| / \sigma_{v}$.

Proof. Divide (17) with (18) to derive the likelihood ratio

$$
\Lambda_{i}\left(\mathbf{G}_{i}\right)=\frac{\operatorname{Pr}\left(\mathbf{G}_{i} \mid \mathbb{H}_{i}\right)}{\operatorname{Pr}\left(\mathbf{G}_{i} \mid \mathbb{H}_{0}\right)}=\prod_{m=1}^{M} e^{\frac{2 \Re\left\{\left(\mathbf{F H}_{t_{i}}\right)^{H} \mathbf{G}_{i}^{(m)}\right\}-\left\|\mathbf{F} \mathbf{H}_{t_{i}}\right\|^{2}}{\sigma_{v}^{2}}}
$$

Taking the logarithm, ignoring the constant terms, and normalizing the result with $2 \sigma_{v}\left\|\mathbf{F H}_{t_{i}}\right\|$, the log likelihood ratio $\ell_{i}\left(\mathbf{G}_{i}\right)=$ $\ln \left(\Lambda_{i}\left(\mathbf{G}_{i}\right)\right)$ simplifies to Eq. (19).

To derive the expression for the mean in Eq. (21), we take the expectation of Eq. (19) as follows.

$$
\mu_{i}=E\left\{\ell_{i}\left(\mathbf{G}_{i}\right) \mid \mathbb{H}_{i}\right\}=\Re\left(\frac{M\left(\mathbf{F H}_{t_{i}}\right)^{H}\left(\mathbf{F} \mathbf{H}_{t_{i}}\right)}{\sigma_{v}\left\|\mathbf{F} \mathbf{H}_{t_{i}}\right\|}\right),
$$

which simplifies to $M\left\|\mathbf{F H}_{t_{i}}\right\| / \sigma_{v}$. The expressions for variances are similarly derived from (19).

Theorem 1 illustrates that the ideal conventional detector is matched to the known signal $\mathbf{F} \mathbf{H}_{t_{i}}$ at the output of the channel, so it is equivalent to a channel matched filter.

\subsection{Unknown Enemy Target Channel Response}

If the channel response $\mathbf{H}_{t_{N-1}}$ for the enemy target is not known, then we use the generalized likelihood ratio (GLR)

$$
\Lambda_{N-1}^{G L R}\left(\mathbf{G}_{i}\right)=\frac{\max _{t_{N-1}}\left\{\operatorname{Pr}\left(\mathbf{G}_{i}\right) \mid \mathbb{H}_{N-1}\right\}}{\operatorname{Pr}\left(\mathbf{G}_{i} \mid \mathbb{H}_{0}\right)}
$$

in the conventional detector. Theorem 2 presents the result.

Theorem 2. The generalized log likelihood ratio $\ell_{N-1}^{G L R}\left(\mathbf{G}_{i}\right)=$ $\ln \left(\Lambda_{N-1}^{G L R}\left(\mathbf{G}_{i}\right)\right)$ for the conventional detector is given by

$$
\ell_{N-1}^{G L R}\left(\mathbf{G}_{i}\right) \mid \mathbb{H}_{N-1}=\sum_{q=0}^{Q-1} \frac{\left|\sum_{m=1}^{M} G_{i}^{(m)}\left(\omega_{q}\right)\right|^{2}}{M \sigma_{v}^{2} / 2}
$$

with the conditional $p d f$

$$
\ell_{N-1}^{G L R}\left(\mathbf{G}_{i}\right) \mid \mathbb{H}_{N-1} \sim \chi_{2 Q}^{2}(\zeta)
$$

where $\zeta=2 M \sum_{q=0}^{Q-1}\left|F\left(\omega_{q}\right)\right|^{2}\left|H_{t_{N-1}}\left(\omega_{q}\right)\right|^{2} / \sigma_{v}^{2}$.

Proof. To derive the target channel response $\mathbf{H}_{t_{N-1}}$ that maximizes $\operatorname{Pr}\left(\mathbf{G}_{i}\right) \mid \mathbb{H}_{N-1}$ in Eq. (23), we take the partial derivative of $\left.\ln \left\{\operatorname{Pr}\left(\mathbf{G}_{i}\right) \mid \mathbb{H}_{N-1}\right)\right\}$, expressed in Eq. (17), with respect to $H_{t_{N-1}}^{*}\left(\omega_{q}\right)$ and set the partial derivative to zero. The estimate for the enemy target response is given by

$$
\widehat{H}_{t_{N-1}}=\mathbf{F}^{-1} \frac{1}{M} \sum_{m=1}^{M} \mathbf{G}_{i}^{(m)} .
$$

Substituting Eq. (26) in Eq. (23), taking the logarithm, normalizing by $\sigma_{v}^{2} / 2$, and ignoring the constant term, the GLR test simplifies to Eq. (24). Next, the statistics for the GLR are shown to be chisquared.

Since the real and imaginary parts of $G_{i}^{(m)}\left(\omega_{q}\right)$ are independent of each other and have the normal pdf $\mathcal{N}\left(F\left(\omega_{q}\right) H_{t_{N-1}}\left(\omega_{q}\right), \sigma_{v} / 2\right)$, each term in Eq. (24) has the following statistics

$$
\frac{\sum_{m=1}^{M} G_{i}^{(m)}\left(\omega_{q}\right)}{\sqrt{M \sigma_{v}^{2} / 2}} \sim \mathcal{C N}\left(\sqrt{2 M / \sigma_{v}^{2}} F\left(\omega_{q}\right) H_{t_{N-1}}\left(\omega_{q}\right), 2\right) .
$$

Since the magnitude of the above terms is the sum of squares of two normal distributions, the GLR has a $\chi$-square distribution with $2 Q$ degrees of freedom. Further, the GLR test in (25) has a noncentral $\chi$-square distribution with $\zeta$ being the noncentral parameter.

\section{N-ARY TIME REVERSAL ML DETECTOR}

Section 4 derives the TR detector for the case when the enemy target response $\mathbf{H}_{t_{N-1}}$ is known as well as the case when $\mathbf{H}_{t_{N-1}}$ is unknown.

\subsection{Known Enemy Target Channel Response}

All the target channel responses, $\mathbf{H}_{t_{i}}$, for $(0 \leq i \leq(N-1))$, are therefore known. In TR, we accumulate the effect of noise $v_{i}$ to $w_{i}$ in the final observation $\mathbf{P}_{i}^{(m)}$. Setting $\sigma_{v}=0$ in Eq. (14), the conditional pdf's of $\mathbf{P}_{i}$ under hypotheses $\mathbb{H}_{i}$ and $\mathbb{H}_{0}$ are given by

$$
\begin{aligned}
& \operatorname{Pr}\left(\mathbf{P}_{i} \mid \mathbb{H}_{i}\right)=\prod_{m=1}^{M} \frac{1}{\pi^{Q}\left(\sigma_{w}^{2}\right)^{Q}} e^{-\frac{\left\|\mathbf{P}_{i}^{(m)}-c\left(\mathbf{F H}_{t_{i}}\right)^{*} \odot \mathbf{H}_{t_{i}}\right\|^{2}}{\sigma_{w}^{2}}} \\
& \operatorname{Pr}\left(\mathbf{P}_{i} \mid \mathbb{H}_{0}\right)=\prod_{m=1}^{M} \frac{1}{\pi^{Q}\left(\sigma_{w}^{2}\right)^{Q}} e^{-\frac{\left\|\mathbf{P}_{i}^{(m)}\right\|^{2}}{\sigma_{w}^{2}}}
\end{aligned}
$$

where all normalization constants $c_{i}$ 's are assumed to be equal to $c$. The log likelihood ratio for time reversal detector is expressed in Theorem 3 .

Theorem 3. The linear test statistics $\ell_{i}\left(\mathbf{P}_{i}\right)$ of the $\boldsymbol{T R}$ detector based on observation vector $\mathbf{P}_{i}$ is given by

$$
\ell_{i}\left(\mathbf{P}_{i}\right)=\Re\left(\frac{\left(\left(\mathbf{F H}_{t_{i}}\right)^{*} \odot \mathbf{H}_{t_{i}}\right)^{H} \sum_{m=1}^{M} \mathbf{P}_{i}^{(m)}}{\sigma_{w}\left\|\left(\mathbf{F} \mathbf{H}_{t_{i}}\right)^{*} \odot \mathbf{H}_{t_{i}}\right\|}\right)
$$

with conditional pdf's

$$
\begin{aligned}
\ell_{i}\left(\mathbf{P}_{i}\right) \mid \mathbb{H}_{0} & \sim \mathcal{C N}(0, M / 2) \\
\ell_{i}\left(\mathbf{P}_{i}\right) \mid \mathbb{H}_{i} & \sim \mathcal{C N}\left(\nu_{i}, M / 2\right)
\end{aligned}
$$

where $\nu_{i}$ is equal to $M c\left\|\left(\mathbf{F H}_{t_{i}}\right)^{*} \odot \mathbf{H}_{t_{i}}\right\| / \sigma_{w}$.

Proof. Divide Eq. (27) by Eq. (28) to obtain the likelihood ratio, take the logarithm, ignore the constant term, and normalize the result with $2 \sigma_{w}\left\|\left(c \mathbf{F H}_{t_{i}}\right)^{*} \odot \mathbf{H}_{t_{i}}\right\|$, lead us to Eq. (29).

To derive the mean $\nu_{i}$, we take the expectation of Eq. (29) as follows

$$
\begin{aligned}
\nu_{i} & =E\left\{\ell_{i}\left(\mathbf{P}_{i}\right) \mid \mathbb{H}_{i}\right\} \\
& =\Re\left(\frac{\left.M c\left(\left(\mathbf{F H}_{t_{i}}\right)^{*} \odot \mathbf{H}_{t_{i}}\right)^{H}\left(\left(\mathbf{F H}_{t_{i}}\right)^{*}\right) \odot \mathbf{H}_{t_{i}}\right)}{\sigma_{w}\left\|\left(\mathbf{F} \mathbf{H}_{t_{i}}\right)^{*} \odot \mathbf{H}_{t_{i}}\right\|}\right)
\end{aligned}
$$

which simplifies to the value of the mean in Eq. (31). 
Since the effect of noise $v_{i}$ is accumulated to $w_{i}$ for the TR detector, we note that the forward reflected signal $\mathbf{G}_{i}^{(m)}$ equals $\mathbf{F H}_{t_{i}}$. Eq. (29) uses both the forward reflected and TR reflected signals. The forward reflected signal $\mathbf{G}_{i}^{(m)}$ is implicit in the $\mathbf{F} \mathbf{H}_{t_{i}}$ term.

\subsection{Unknown Enemy Target Channel Response}

For the TR detector, we stack the conjugate of the forward observations (Eq. (11)) with the TR observations (Eq. (12)) in $(2 Q M \times 1)$ vector $\mathbf{R}_{i}$ as follows

$$
\begin{aligned}
\mathbf{R}_{\mathbf{i}} & =\left[\begin{array}{c}
\mathbf{G}_{i}^{*} \\
\mathbf{P}_{i}
\end{array}\right] \\
& =\left[\begin{array}{c}
\mathbf{1} \otimes\left(\mathbf{F} \mathbf{H}_{t_{i}}\right)^{*} \\
\mathbf{c}_{\mathbf{i}} \otimes\left[\mathbf{F}^{*} \mathbf{H}_{t_{i}}^{*} \odot \mathbf{H}_{t_{i}}\right]
\end{array}\right]+\left[\begin{array}{c}
\mathbf{V}_{i}^{*} \\
\left(\mathbf{c}_{\mathbf{i}} \otimes \mathbf{H}_{t_{i}}\right) \odot \mathbf{V}_{i}^{*}+\mathbf{W}_{i}
\end{array}\right] .
\end{aligned}
$$

In terms of $\mathbf{R}_{\mathbf{i}}$, the GLR test for the TR detector is given by

$$
\Lambda_{N-1}^{G L R}\left(\mathbf{R}_{i}\right)=\frac{\max _{t_{N-1}}\left\{\operatorname{Pr}\left(\mathbf{R}_{i} \mid \mathbb{H}_{N-1}\right)\right\}}{\operatorname{Pr}\left(\mathbf{R}_{i} \mid \mathbb{H}_{0}\right)}
$$

To save on space, we express $F\left(\omega_{q}\right)$ as $F, G_{i}\left(\omega_{q}\right)$ as $G_{i}$, and $\widehat{H}_{t_{N-1}}\left(\omega_{q}\right)$ as $\widehat{H}_{t_{N-1}}$ in the following theorem.

Theorem 4. The generalized log likelihood ratio test $\ell_{N-1}^{G L R}\left(\mathbf{R}_{i}\right)$ for the TR detector is given by

$$
\begin{aligned}
& \ell_{N-1}^{G L R}\left(\mathbf{R}_{i} \mid \mathbb{H}_{N-1}\right)= \\
& \quad \frac{1}{\sigma_{v}^{2} \sigma_{w}^{2}} \sum_{m=1}^{M} \sum_{q=0}^{Q-1}\left[\left\{\sigma_{v}^{2} c_{i}^{(m)^{2}}\left|G_{i}^{(m)}\right|^{2}+\sigma_{w}^{2}|F|^{2}\right\}\left|\widehat{H}_{t_{N-1}}\right|^{2}\right. \\
& \left.\quad-2 \Re\left\{\left(c_{i}^{(m)} P_{i}^{(m)^{*}} G_{i}^{(m)^{*}} \sigma_{v}^{2}+G_{i}^{(m)^{*}} F \sigma_{w}^{2}\right) \widehat{H}_{t_{N-1}}\right\}\right]
\end{aligned}
$$

Proof. Using Eq. (32), the statistics of $\left(\mathbf{R}_{\mathbf{i}} \mid \mathbb{H}_{\mathbb{N}}\right)$ is given by

$$
\operatorname{Pr}\left(\mathbf{R}_{\mathbf{i}} \mid \mathbb{H}_{N-1}\right)=\frac{1}{\sqrt{(2 \pi)^{2 Q M}|\mathbf{C}|}} \exp \left\{-\frac{1}{2} \mathbf{X}_{\mathbf{i}}{ }^{H} \mathbf{C}^{-1} \mathbf{X}_{\mathbf{i}}\right\}
$$

where $\mathbf{X}_{\mathbf{i}}$ is the mean vector of $\mathbf{R}_{\mathbf{i}}$ and $\mathbf{C}$ is the covariance matrix of $\mathbf{R}_{\mathbf{i}}$ in Eq. (32). The covariance matrix is given by

$$
\mathbf{C}=\left[\begin{array}{cc}
\sigma_{v}^{2} \mathbf{I}_{M} \otimes \mathbf{I}_{Q} & \sigma_{v}^{2} \mathbf{c}_{\mathbf{i}} \otimes \mathbf{H}_{t_{i}}^{*} \\
\sigma_{v}^{2} \mathbf{c}_{\mathbf{i}} \otimes \mathbf{H}_{t_{i}} & \sigma_{v}^{2} \mathbf{C}_{\mathbf{i}}{ }^{2} \otimes \operatorname{diag}\left[\mathbf{H}_{t_{i}}^{*} \odot \mathbf{H}_{t_{i}}\right]+\sigma_{w}^{2} \mathbf{I}_{M} \otimes \mathbf{I}_{Q}
\end{array}\right]
$$

When no target is present, the $\operatorname{Pr}\left(\mathbf{G}_{\mathbf{i}} \mid \mathbb{H}_{0}\right)$ and $\operatorname{Pr}\left(\mathbf{P}_{\mathbf{i}} \mid \mathbb{H}_{0}\right)$ are independent, so the $\operatorname{Pr}\left(\mathbf{R}_{\mathbf{i}} \mid \mathbb{H}_{0}\right)$ is simply the multiplication of Eq. (18) and Eq. (28).

To estimate $H_{t_{N-1}}\left(\omega_{q}\right)$ that maximizes $\operatorname{Pr}\left(\mathbf{R}_{i} \mid \mathbb{H}_{N-1}\right)$, we take the partial derivative of $\ln \left\{\operatorname{Pr}\left(\mathbf{R}_{\mathbf{i}} \mid \mathbf{H}_{t_{N-1}}\right)\right\}$ with respect to $H_{t_{N-1}}^{*}\left(\omega_{q}\right)$. Ignoring the constant terms, the result simplifies to

$\widehat{H}_{t_{N-1}}=\frac{\sum_{m=1}^{M}\left[\frac{G_{i}^{(m)}\left(\omega_{q}\right) F\left(\omega_{q}\right)^{*}}{\sigma_{v}^{2}}+\frac{c_{i}^{(m)} G_{i}^{(m)}\left(\omega_{q}\right) P_{i}^{(m)^{*}}\left(\omega_{q}\right)}{\sigma_{w}^{2}}\right]}{\sum_{m=1}^{M}\left[\frac{\left|F\left(\omega_{q}\right)\right|^{2}}{\sigma_{v}^{2}}+\frac{\left|G_{i}^{(m)}\left(\omega_{q}\right)\right|^{2} c_{i}^{(m)^{2}}}{\sigma_{w}^{2}}\right]}$

The expression for the GLR test is derived by taking the logarithm of the ratio of $\operatorname{Pr}\left(\mathbf{R}_{\mathbf{i}} \mid \mathbb{H}_{N-1}\right)$ and $\operatorname{Pr}\left(\mathbf{R}_{\mathbf{i}} \mid \mathbb{H}_{0}\right)$ evaluated at the maximum likelihood estimate of $\widehat{H}_{t_{N-1}}\left(\omega_{q}\right)$.
In the conventional and TR detectors, the values of the thresholds $\eta_{i}$ are obtained by fixing the probability of false alarm $P_{F}$, which is defined as

$$
P_{F}=\int \ldots \int_{\mathbf{z}} \operatorname{Pr}\left(\ell_{1}, \ldots, \ell_{N-1} \mid \mathbb{H}_{0}\right) d \ell_{1} \ldots d \ell_{N-1}
$$

where $\mathbf{Z}=\mathbf{Z}_{1} \cup \ldots \cup \mathbf{Z}_{N-1}$ with $\mathbf{Z}_{i}$ being the observation space associated with hypothesis $\mathbb{H}_{i}$ corresponding to the presence of target $i$. It is difficult to derive a simplified analytical expression for the probability of false alarm for a general $N$-ary detection problem. The specialized case of $N=3$ is considered in the experimental section, where the simplified expression for the probability of false alarm is derived.

\section{EXPERIMENTS}

To test the performance of our detection algorithms, we simulate a 3 -ary detection problem corresponding to detecting the presence of one of the two possible targets and distinguishing one target from the other. Such a scenario is often encountered in defence applications where the enemy target $(i=2)$ needs to be distinguished from the friendly target $(i=1)$. Using the Neyman Pearson test, the 3-ary conventional detector is given by

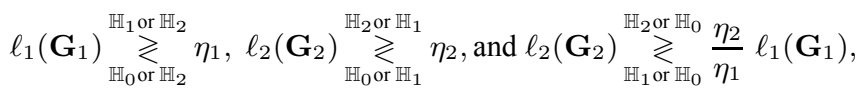

where thresholds $\eta_{1}$ and $\eta_{2}$ are obtained by fixing the probability of false alarm

$$
\begin{aligned}
& P_{F}=\frac{3}{4}-\frac{1}{4}\left[\operatorname{erf}\left(\frac{\eta_{1}}{\sqrt{M}}\right)-\operatorname{erf}\left(\frac{\eta_{2}}{\sqrt{M}}\right)-\operatorname{erf}\left(\frac{\eta_{1}}{\sqrt{M}}\right) \operatorname{erf}\left(\frac{\eta_{2}}{\sqrt{M}}\right)\right] \\
& \text { with } \quad \operatorname{erf}(x)=\frac{2}{\sqrt{\pi}} \int_{0}^{x} e^{-t^{2}} d t, \quad x \in \mathbb{R},
\end{aligned}
$$

to 0.01 in our simulations. The likelihood ratio $\ell_{2}$ for the enemy target with an unknown signature is derived from the GLR test (Eq. (24) for the conventional detector and Eq. (34) for the TR detector), while the likelihood ratio $\ell_{1}$ for the friendly target assumes a known signature and is based on the standard likelihood ratio test (Eq. (19) for the conventional detector and Eq. (29) for the TR detector).

The 3-ary TR detector uses the same likelihood expressions, Eq. (37), except that test statistics $\ell_{1}\left(\mathbf{P}_{1}\right)$ and $\ell_{2}\left(\mathbf{P}_{2}\right)$ are used instead. In our simulations, the propagation medium has dimensions of $R \in(350 \times 200) \lambda$, with the transducer element located at $(220,150) \lambda$. The medium has a random velocity profile having a Gaussian distribution with a standard deviation of $10 \%$ about the mean value of $c_{0}=1500 \mathrm{~m} / \mathrm{s}$. Randomness in the velocity profile leads to multiple paths because of total internal reflections. An ensemble of the velocity profile is shown in Fig. 1. The probing pulse is given by

$$
f(t)=-2 \pi^{2} v^{2}(t-1 / v) e^{-\pi^{2} v^{2}(t-1 / v)^{2}},
$$

for $(0 \leq t \leq 0.7 \mu \mathrm{s})$, and the central frequency $v$ equals $3 \mathrm{MHz}$. The probing pulse propagates the random medium according to the wave hydrodynamic equation

$$
\Delta \psi(\vec{y}, t)-\frac{1}{(c(\vec{x}))^{2}} \frac{\partial^{2} \psi(\vec{y}, t)}{\partial t^{2}}=-f(t) \delta\left(\vec{y}-\vec{y}_{p}\right) .
$$

The grid size is $(0.25 \lambda \times 0.25 \lambda)$ so the discretized domain has dimensions of $(881 \times 601)$ and the value of wavelength $\lambda$ is 


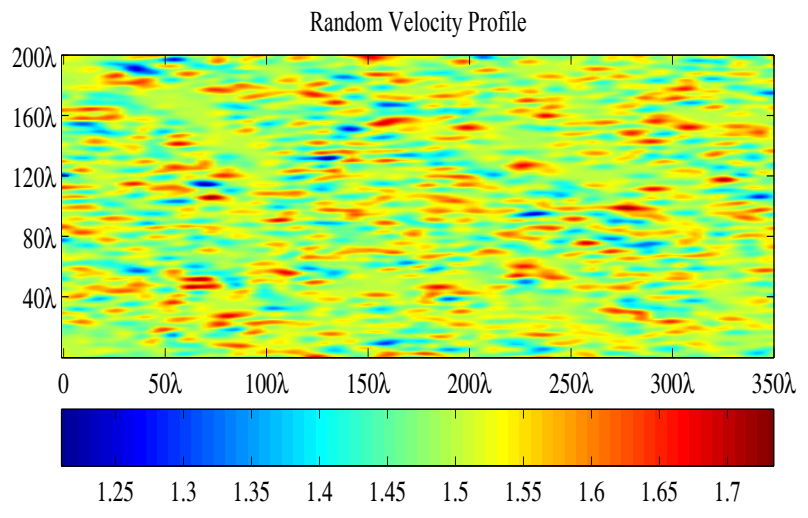

Fig. 1. Random realization of the velocity profile $c(\vec{x})$.

$0.5 \mathrm{~mm}$. Based on the CFL conditions, the time step $\Delta t$ is set to $5 \times 10^{-8} \mathrm{~s}$. Two different targets are simulated by associating different frequency characteristics to each target. The channel frequency responses for the two targets are plotted in Fig. 2 as a function of the angular frequency $\omega$. Details of the setup are given in [4].

To simulate the effect of observation noise, additive White Gaussian noise is added to the final observations, $g_{i}\left[\vec{y}_{p}, \vec{y}_{t_{i}}, k\right]$ for the conventional detector and $p_{i}\left[\vec{y}_{p}, \vec{y}_{t_{i}}, k\right]$ for the TR detector. The variance of the noise is changed to account for different SNR's. Fig. 3 illustrates the results of the Monte Carlo simulations plotting the receiver operating characteristics (ROC) for the enemy target (top) and friendly target (bottom) as functions of the probabilities of detection $P_{D}$ versus the SNR's. The ROC's for the TR detector are plotted using symbol $\circ$, while the ROC's for the conventional detector are shown with symbol $\times$. For both targets, the TR detector outperforms the conventional detector. At $P_{D}=0.5$, for example, the TR detector provides a gain of over $5 \mathrm{~dB}$ with respect to the conventional detector. Similar improvements are observed for other performance statistics.

\section{SUMMARY}

The paper designs an $\mathrm{N}$-ary time reversal detector capable of detecting the presence of a target and classifying the detected target as an unknown enemy target or one of the $(N-2)$ known friendly targets. Compared to the conventional detectors, which use only the forward propagation path and do not include the time reversal step, the TR detector provides a gain of about $5 \mathrm{~dB}$ at low SNR's.

\section{REFERENCES}

[1] M. Fink, "Time Reversal of Ultrasonic Fields - Part I: Basic Principles," IEEE Trans. on Ultrasonics, Ferroelectrics, and Freq. Control, vol. 39, 1992, pp. 555-566.

[2] E.J. Sullivan and D. Middleton, "Estimation and Detection Issues in Matched Field Processing," IEEE Trans. on Ocean Engineering, vol. 18, 1993, pp. 156-167.

[3] J. M. F. Moura and Y. Jin, "Detection by time reversal: single antenna,"IEEE Trans. on Signal Processing, vol. 55, 2007, pp. $187-201$

[4] A. Asif, Q. Bai, and J. M. F. Moura, "Time Reversal Matched Field Processing: An Analytical Justification," in Proceedings of ISSPIT, Vancouver, 2006.
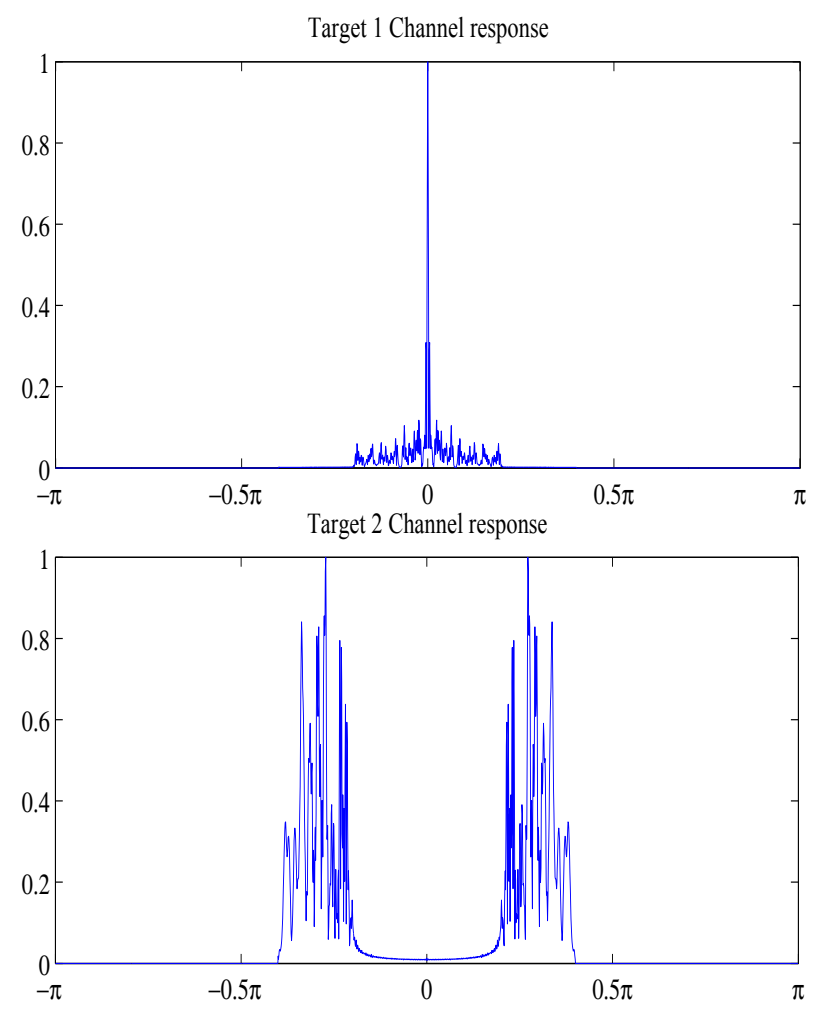

Fig. 2. Channel responses: Target 1 (top) and Target 2 (bottom).

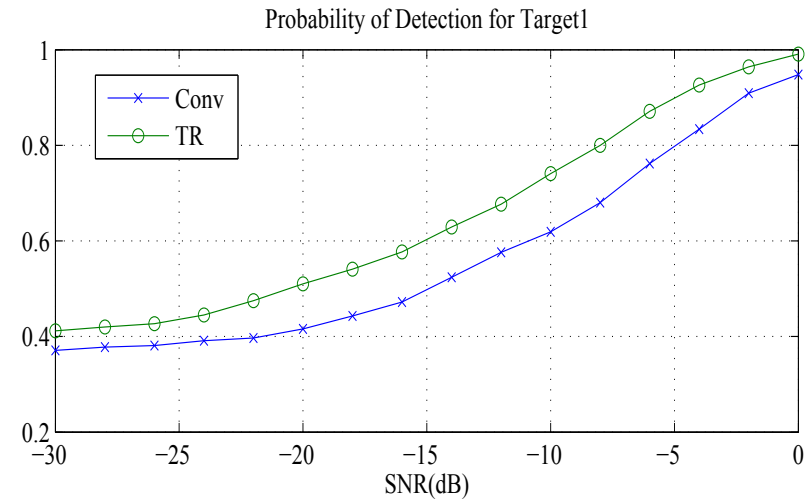

Probability of Detection for Target 2

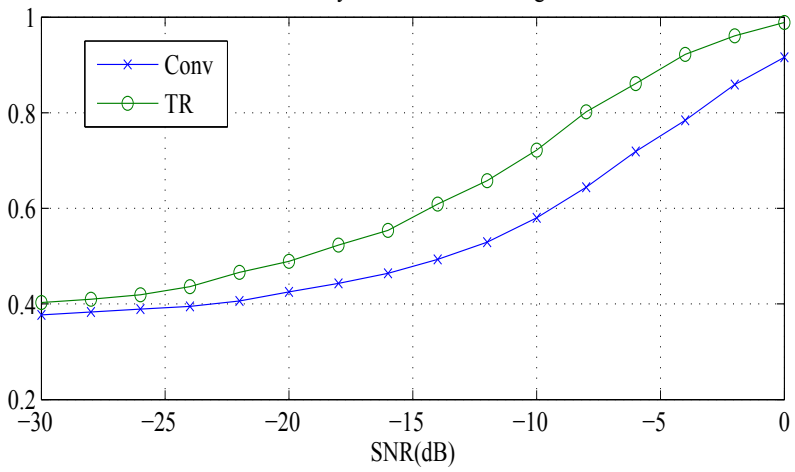

Fig. 3. Detection probabilities: Target 1 (top) and 2 (bottom). 